\title{
A Survey: Image Segmentation Techniques
}

\author{
Muhammad Waseem Khan
}

\begin{abstract}
Segmentation is considered as one of the main steps in image processing. It divides a digital image into multiple regions in order to analyze them. It is also used to distinguish different objects in the image. Several image segmentation techniques have been developed by the researchers in order to make images smooth and easy to evaluate. This paper presents a literature review of basic image segmentation techniques from last five years. Recent research in each of image segmentation technique is presented in this paper.
\end{abstract}

Index Terms-Fuzzy theory, PDE based image segmentation, segmentation, threshold.

\section{INTRODUCTION}

Famous techniques of image segmentation which are still being used by the researchers are Edge Detection, Threshold, Histogram, Region based methods, and Watershed Transformation. Since images are divided into two types on the basis of their color, i.e. gray scale and color images. Therefore image segmentation for color images is totally different from gray scale images, e.g., content based image retrieval[1], [2]. Also which algorithm is robust and works well is depends on the type of image [3]. The property of a pixel in an image and information of pixels near to that pixel are two basic parameters for any image segmentation algorithm. It can also be representing as similarity of pixels in any region and discontinuity of edges in image. Edge based segmentation is used to divide image on the basis of their edges. Region based methods used the threshold in order to separate the background from an image, whereas neural network based techniques used the learning algorithm to train the image segmentation process [4]. The result taken from image segmentation process is the main parameter for further image processing research; this result will also determine the quality of further image processing process. Image segmentation algorithms play an important role in medical applications, i.e., diagnosis of diseases related to brain [5]-[8] heart, knee, spine, pelvis, prostate and blood vessel, and pathology localization. Therefore, Image segmentation is still a very hot area of research for image processing field. It is still a challenging task for researchers and developers to develop a universal technique for image segmentation [9]. Image segmentation is also used to differentiate different objects in the image, since our image is divided into foreground and background, whereas foreground of image is related to the region of interest, and background is the rest of the image. Hence, image segmentation will separate these two parts from one another. Therefore, the main three

Manuscript received August 28, 2013; revised October 30, 2013.

The author is with the COMSATS Institute of Information Technology, Pakistan (e-mail: Muhammad.wasim1@gmail.com). approaches for image segmentation are, Threshold, Edge, and Region based [10].

\section{LITERATURE REVIEW OF IMAGE SEGMENTATION TECHNIQUES}

All basic image segmentation techniques currently being used by the researchers and industry will be discussed and evaluate in this section.

\section{A. Edge Based Image Segmentation}

Fernando C. Monteiro [11] proposed a new image segmentation method comprises of edge and region based information with the help of spectral method and morphological algorithm of watershed. Firstly, they reduce the noise from image using bilateral filter as a pre-processing step, secondly, region merging is used to perform preliminary segmentation, region similarity is generated and then graph based region grouping is perform using Multi-class Normalized Cut method [12]. Berkley segmentation dataset is use as a dataset. They compare the technique with mean shift, multi-scale graph based segmentation, and JSEG. It is found that proposed technique has outperformed other methods and produce better results.

R. V. Patil [13] claims that if the number of clusters is estimated in accurate manner, K-means image segmentation will provide better results. They proposed a new method based on edge detection to estimate number of clusters. Phase congruency is used to detect the edges. Then these edges are used to find clusters. Threshold and Euclidean distance is used in order to make clusters. K-means is sued to find the final segmentation of image. MATLAB is used to implement the proposed technique. Experiments are performed on nine different images and results shows that number of clusters is accurate and optimal. Weihong Cui Yi Zhang [14] proposed an edge based auto threshold select method to generate multi-scale image segmentation. Band weight and NDVI (Normalized Difference Vegetation Index) is used to calculate edge weight. MST and Edge based Threshold method is used to perform image segmentation. Experiments are performs on multi-scale resolution images, i.e., Quick-bird multispectral images. Results have shown that their method maintain the object information and keep object boundaries while segment the image. Anna Fabijańska [15] introduced a new method uses Variance Filter for edge detection in image segmentation process. Their method found the edge position using Variance Filter. Sobel Gradient filter with K-means is also used to extract the edges and compared with the proposed technique. The effect of filtering window size on determining edges is also discussed and it is found that if the $9 \times 9$ window is used to extract edges then edge is complete accurately match the shape of object in the 
image. In case of larger details images, a small filtering window is proffered. Results have shown that their proposed technique outperform Sobel Edge Detector.

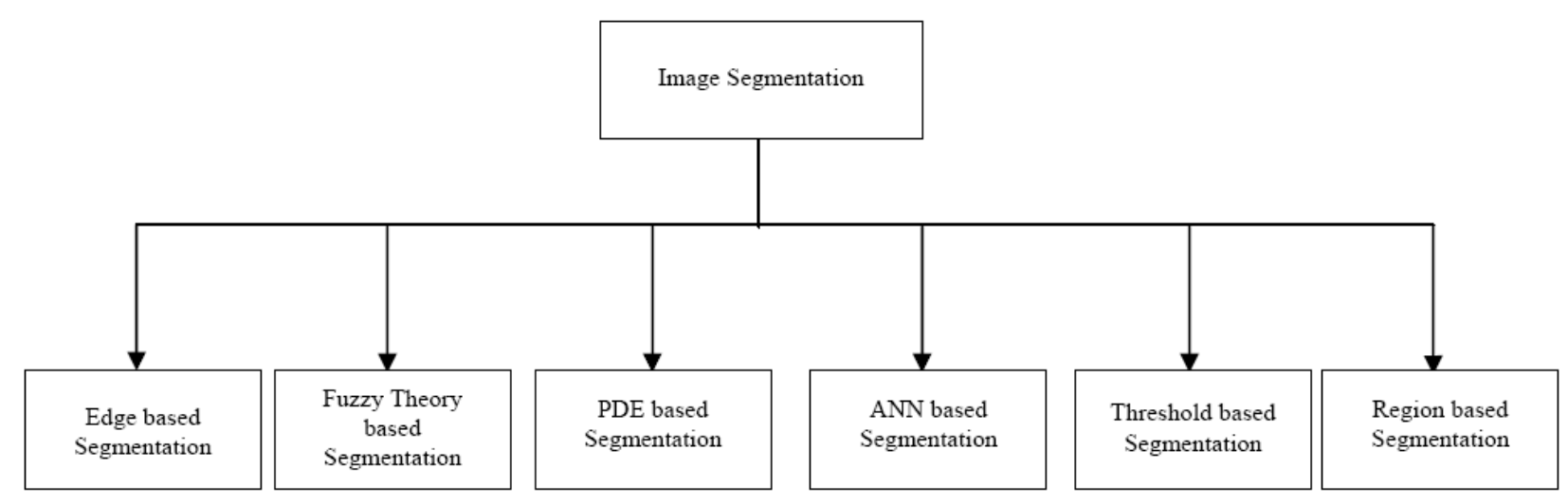

Fig. 1. Image segmentation techniques

Mohammed J. Islam [16] found that Computer Vision is a best solution for real time inspection of capsules in pharmaceutical industry. Author has developed a system for quality inspection using edge based image segmentation techniques [17]. They used Sobel Edge Detector [18] in order to detect edges with noise-suppression property. After edge detection, Otsu Thresholding technique is used for localization of background and foreground pixels. Experiments are conducted and results are compared with NN-based segmentation technique building Visual $\mathrm{C}++$. Results outperform NN technique on the basis of accuracy and processing time difference of $10 \mathrm{~ms}$.

\section{B. Fuzzy Theory Based Image Segmentation}

Liu Yucheng [19] proposed a new fuzzy morphological based fusion image segmentation algorithm. Algorithm has used morphological opening and closing operations to smooth the image and then perform the gradient operations on the resultant image [20]. After compare the proposed fusion algorithm with Watershed algorithm[21] and Prewitt methods, it is found that fusion approach solve the problem of over-segmentation of Watershed algorithm. It also save the information details of image and improve the speed as well. Syoji Kobashi [22] used scale based fuzzy connected image segmentation and fuzzy object model to segment the cerebral parenchyma region of new born brain MRI image. Foreground region is separated in first step, correction of MRI intensity in-homogeneity is performed next, and then scale-base Fuzzy Object Model (FOM) is applied on resultant image. Results of proposed method are evaluated on the basis of Fast Positive Volume Fraction (FPVF) and Fast Negative Volume Fraction (FVNF). Results from experiment have shown that FOM (Fuzzy object model) has attained minimum FPVF and FVNF values. Refik Samet [23] proposed a new Fuzzy Rule based image segmentation technique to segment the rock thin segment images. They take RGB image of rock thin segment as input and give segmented mineral image as output. Fuzzy C Means is also applied on rock thin images and results are compared of both techniques. Firstly, the user will take sample image from minerals; features are distinguished on the basis of red, green and blue components of image. Membership function is defined for each component using Fuzzy rules. Each membership function represents the color's distribution in the image. Strong and weak points are defined, whereas strong points are considered as seed points and weak points become their members. Results have shown that proposed technique is better than FCM algorithm. Muhammad Rizwan Khokher [24] presented a new method of image segmentation using Fuzzy Rule based system and Graph Cuts. Authors have firstly segmented the gray scale, color, and texture images using Graph Cuts. Weights are assigned to the features of image using Fuzzy Rules. Their algorithm works by firstly extracting the features of image, calculate the constants using fuzzy rules, calculate the weighted average of constants to find the similarity matrix, partition the graph using Normalized Graph Cut method[25], and finally get the segmented image from partitioned graph. Berkley database is used to evaluate the algorithm. Simulation is performed in Matlab and C language. Results are evaluated on the basis of Mean, Standard Deviation, and PPV value. It is found that most of the evaluation measure range from 0.85 to 0.95 for S.D and PPV.

\section{Partial Differential Equation (PDE) Based Image Segmentation}

Jinsheng Xiao [26] proposed a new non-linear discontinue partial differential equation (PDE) that models the level set method of gray images. A discrete method is also proposed to find numerical solution and to implement the filter. Non-Linear discontinue PDE formula is applied on image of cameramen using MATLAB. Results have shown that image edges and boundaries are remained blurred and can be shifted by using Close operator. More information can be saved by using the proposed scheme. Fengchun Zhang [27] presents a variation model using 4 th order PDE with $2^{\text {nd }}$ order PDE for finger vein image de-noising. Midpoint Threshold segmentation technique is used to extract the region of interest accurately. $4^{\text {th }}$ order PDE has reduced the noise very well, whereas $2^{\text {nd }}$ order PDE has approximated the boundaries effectively. It can be observed from experiments that PSNR value of proposed method is increase by $2 \mathrm{~dB}$. Method is compared with threshold based segmentation algorithm and it is found that proposed method has segment the real finger vein image accurately. Chun Yuan[28] proposed a new segmentation model for color images. Their 
model is based on Geodesic Active Contour (GAC) model. But GAC is only restricted to gray scale images. Therefore their model is also an extension of GAC model, and known as color-GAC model. It uses the expression of the Gradient of color image.

\section{Artificial Neural Network (ANN) Based Image Segmentation}

Wencang Zhao [29] proposed a new image segmentation algorithm based on textural features[30] and Neural Network[31] to separate the targeted images from background. Dataset of micro-CT images are used. De-noising filter is used to remove noise from image as a pre-processing step, Feature extraction is performed next, and then Back Propagation Neural Network is created, and lastly, it modifies the weight number of network, and save the output. Proposed algorithm is compared with Thresholding method and Region Growing method. Results have shown that proposed technique outperforms other methods on the basis of speed and accuracy of segmentation. Lijun Zhang [32] proposed a new neural network based image segmentation system for color images. They combined the Wavelet Decomposition and Self Organizing Map (SOM) to propose a new method, i.e., SOM-NN. Voting among child pixels selected the parent pixel. After initialization, ANN found the segmentation result which satisfies all levels. Wavelet decomposition is performed to remove noise. Hence wavelet decomposition and SOM-NN are combined to perform segmentation. Results have shown that method has reduce noise and produce accurate segmentation. Shohel Ali Ahmed [33] proposed Image Texture Classification technique based on Artificial Neural Networks (ANN). Firstly, image is captured and pre-processing is performed, after it, feature extraction[34] is performed, whereas, ANN classifier [35] is used for texture classification, Clustering is performed to separates background from sub-images. Trained ANN combines the input pixels into two clusters which give results. It produces the texture classification and segmentation of image.

\section{E. Threshold Based Image Segmentation}

Shiping Zhu [36] proposed a new threshold based edge detection and image segmentation algorithm. They calculate the threshold of each pixel in the image on the basis of its neighboring pixels. They also find the edges of image with the help of proposed algorithm. A threshold of each pixel was set using histogram. PDF is used to isolate the background and threshold of image. They implement their algorithm in Visual C++. Results outperform the Canny Operator results, because it performs edge detection and segmentation simultaneously. Anping XU [37] proposed a threshold-based level set approach comprising both threshold based segmentation and Fast Marching Method (FMM) for medical image segmentation [38]. The result of de-noising filter is passed to FMM for segmentation purpose with the help of threshold based level set technique. They implement their method in $\mathrm{VC}++$ and ITK. After the experiment, results have shown that level set method based on threshold results in clearer, accurate and more perfect segmentation, it also avoid from edge leakage and improve the speed of segmentation.
Wu Kaihua and Ban Tao [39] have presented a new optimal threshold segmentation method based on entropy criteria and Genetic algorithm in order to improve the image acquisition process in computer vision. The factors taken by them are illumination, light, reflection of light, CCD exposure time and some properties of image histogram. They compare their proposed technique with Otsu algorithm and found that their algorithm is efficient in searching and in finding threshold based segmentation of an image. Frank Jiang [40] proposed a new multilevel Threshold-based segmentation technique using PSO and Wavelet mutation. They also proposed a new PSO algorithm which is used in first two steps of algorithm. Then the output of PSO is passed to wavelet mutation operations which performs the mutation operation and update the PSO equations after it. This work will generate optimized threshold and correct segmentation. After comparing their method with HCOCLPSO, they found that it produces optimal threshold as compare to other method. They claim that their algorithm is best for real time applications, e.g., error resilient video application in hostile environment.

\section{F. Region Based Image Segmentation}

D. Barbosa [41] proposed a new image segmentation technique which joins the edge and region based information with spectral method using Morphological Watershed algorithms. Firstly noise filter is used with Magnitude Gradient in a pre-processing stage, secondly, pre-segmentation is done using region merging, then region similarity graph is generated and finally segmentation is performed using Multi Class Normalized Cut. Method is compared with Mean Shift, MNCUT, and JSEG using natural images. Proposed technique overcomes Spectral Clustering method. Gang Chen [42] found that fast extraction of object information from a given image is still a problem for real time image processing. They also found that region based methods are also time consuming and not give effective segmentation. They proposed a new region based method based on Least Square method in order to detect objects sharply. They used a weight matrix for region based method which also takes the local information into account and also the usage of Least Square method provides optimal and fast segmentation. Comparison of their method is conducted with Otsu method and Chan-Vese method using Lena image. Their method can extract the features more accurately than other methods. Zhen Hua, Yewei Li [43] proposed a new image segmentation method based on improved visual attention and region growing approach. The gray values and edges of input image are extracted using Gabor filter [44] and Guass-Laplace filters [45]. Then ANN methods are used to extract the region of interest. Experiments are conducted on natural scene images, and it is found that their algorithm not only segmented the image perfectly but also found the salience edges which others can't. Tiancan Mei [46] claims that Markov random field (MRF) [47] is suffered from lack of handling the large range of interaction. In order to overcome this drawback of MRF, they presented a new supervised image segmentation method, i.e., Region based Multi-scale segmentation method. Natural scene images are used as a 
dataset. By using region as a parameter in Multi-scale MRF model, their algorithms perform better than other techniques. It is observed from results that RSMAP improve the MSAP method used for image segmentation.

\section{CONCLUSION}

In this paper, we discuss and evaluate main image segmentation techniques used for the purpose of image analysis. It is found that there is no perfect method for image segmentation because the result of image segmentation is depends on many factors, i.e., pixel color, texture, intensity, similarity of images, image content, and problem domain. Therefore, it is not possible to consider a single method for all type of images nor all methods can perform well for a particular type of image. Hence, it is good to use hybrid solution consists of multiple methods for image segmentation problem.

\section{REFERENCES}

[1] M. Yasmin, S. Mohsin, I. Irum, and M. Sharif, "Content based image retrieval by shape, color and relevance feedback," Life Science Journal, vol. 10, 2013.

[2] M. Rehman, M. Iqbal, M. Sharif, and M. Raza, "Content based image retrieval: survey," World Applied Sciences Journal, vol. 19, pp. 404-412, 2012.

[3] M. M. S. J. Preetha, L. P. Suresh, and M. Bosco, "Image segmentation using seeded region growing," in Proc. International Conference on Computing, Electronics and Electrical Technologies (ICCEET), pp. 576-583, 2012.

[4] W. X. Kang, Q. Q. Yang, and R. P. Liang, "The comparative research on image segmentation algorithms," in Proc. First International Workshop on Education Technology and Computer Science, 2009. ETCS'09. pp. 703-707, 2009.

[5] M. Yasmin, S. Mohsin, M. Sharif, M. Raza, and S. Masood, "Brain Image Analysis: A Survey," World Applied Sciences Journal, vol. 19, pp. 1484-1494, 2012.

[6] S. Masood, M. Sharif, M. Yasmin, M. Raza, and S. Mohsin, "Brain Image Compression: A Brief Survey," Research Journal of Applied Sciences, vol. 5, 2013.

[7] M. Yasmin, M. Sharif, S. Masood, M. Raza, and S. Mohsin, "Brain image reconstruction: A short survey," World Applied Sciences Journal, vol. 19, pp. 52-62, 2012.

[8] M. Raza, M. Sharif, M. Yasmin, S. Masood, and S. Mohsin, "Brain image representation and rendering: A survey," Research Journal of Applied Sciences, vol. 4, 2012.

[9] T. Chen and Z. Shen, "An adaptive image segmentation method using region growing," in Proc. 2nd International Conference on Computer Engineering and Technology (ICCET), vol. 7, pp. 78-80, 2010

[10] H. Hedberg, "A survey of various image segmentation techniques," Dept. of Electroscience, Box, vol. 118, 2010

[11] F. C. Monteiro and A. Campilho, "Watershed framework to region-based image segmentation," in Proc. International Conference on Pattern Recognition, ICPR 19th, pp. 1-4, 2008.

[12] M. Hameed, M. Sharif, M. Raza, S. W. Haider, and M. Iqbal, "Framework for the comparison of classifiers for medical image segmentation with transform and moment based features," Research Journal of Recent Sciences, vol. 2277, p. 2502, 2012

[13] R. Patil and K. Jondhale, "Edge based technique to estimate number of clusters in k-means color image segmentation," in Proc. 3rd IEEE International Conference on Computer Science and Information Technology (ICCSIT), pp. 117-121, 2010.

[14] W. Cui and Y. Zhang, "Graph based multispectral high resolution image segmentation," in Proc. International Conference on Multimedia Technology (ICMT), pp. 1-5, 2010.

[15] A. Fabijanska, "Variance filter for edge detection and edge-based image segmentation," in Proc. International Conference on Perspective Technologies and Methods in MEMS Design (MEMSTECH), pp. 151-154, 2011.

[16] M. J. Islam, S. Basalamah, M. Ahmadi, and M. A. S. hmed, "Capsule image segmentation in pharmaceutical applications using edge-based techniques," IEEE International Conference on Electro/Information Technology (EIT), pp. 1-5, 2011.

[17] M. SHARIF, M. RAZA, and S. MOHSIN, "Face recognition using edge information and DCT," Sindh Univ. Res. Jour. (Sci. Ser.), vol. 43, no. 2, pp. 209-214, 2011

[18] W. Haider, M. S. Malik, M. Raza, A. Wahab, I. A. Khan, U. Zia, J. Tanveer, and H. Bashir, "A hybrid method for edge continuity based on Pixel Neighbors Pattern Analysis (PNPA) for remote sensing satellite images," Int'l J. of Communications, Network and System Sciences, vol. 5, pp. 624-630, 2012.

[19] L. Yucheng and L. Yubin, "An algorithm of image segmentation based on fuzzy mathematical morphology," in International Forum on Information Technology and Applications, IFITA'09, pp. 517-520, 2009.

[20] W. Haider, M. Sharif, and M. Raza, "Achieving accuracy in early stage tumor identification systems based on image segmentation and 3D structure analysis," Computer Engineering and Intelligent Systems, vol. 2, pp. 96-102, 2011.

[21] M. S. A. Shahzad, M. Raza, and K. Hussain, "Enhanced watershed image processing segmentation," Journal of Information \& Communication Technology, vol. 2, pp. 1-9, 2008.

[22] S. Kobashi and J. K. Udupa, "Fuzzy object model based fuzzy connectedness image segmentation of newborn brain MR images," in Proc. IEEE International Conference on Systems, Man, and Cybernetics (SMC), pp. 1422-1427, 2012.

[23] R. Samet, S. E. Amrahov, and A. H. Ziroglu, "Fuzzy rule-based image segmentation technique for rock thin section images," in Proc. 3rd International Conference on Image Processing Theory, Tools and Applications (IPTA), pp. 402-406, 2012.

[24] M. R. Khokher, A. Ghafoor, and A. M. Siddiqui, "Image segmentation using fuzzy rule based system and graph cuts," in Proc. 12th International Conference on Control Automation Robotics \& Vision (ICARCV), pp. 1148-1153, 2012.

[25] M. Sharif, S. Mohsin, M. J. Jamal, and M. Raza, "Illumination normalization preprocessing for face recognition," in Proc. International Conference on Environmental Science and Information Application Technology (ESIAT), pp. 44-47, 2010.

[26] J. Xiao, B. Yi, L. Xu, and H. Xie, "An image segmentation algorithm based on level set using discontinue PDE," in Proc. First International Conference on Intelligent Networks and Intelligent Systems, ICINIS'08., pp. 503-506, 2008.

[27] F. Zhang, S. Guo, and X. Qian, "Segmentation for finger vein image based on PDEs denoising," in Proc. 3rd International Conference on Biomedical Engineering and Informatics (BMEI), pp. 531-535, 2010.

[28] C. Yuan and S. Liang, "Segmentation of color image based on partial differential equations," in Proc. Fourth International Symposium on Computational Intelligence and Design (ISCID), pp. 238-240, 2011.

[29] W. Zhao, J. Zhang, P. Li, and Y. Li, "Study of image segmentation algorithm based on textural features and neural network," in International Conference on Intelligent Computing and Cognitive Informatics (ICICCI), pp. 300-303, 2010.

[30] M. Sharif, M. Y. Javed, and S. Mohsin, "Face recognition based on facial features," Research Journal of Applied Sciences, Engineering and Technology, vol. 4, pp. 2879-2886, 2012.

[31] M. Yasmin, M. Sharif, and S. Mohsin, "Neural networks in medical imaging applications: A survey," World Applied Sciences Journal, vol. 22, pp. 85-96, 2013.

[32] L. Zhang and X. Deng, "The research of image segmentation based on improved neural network algorithm," in Proc. Sixth International Conference on Semantics Knowledge and Grid (SKG), pp. 395-397, 2010.

[33] S. A. Ahmed, S. Dey, and K. K. Sarma, "Image texture classification using Artificial Neural Network (ANN)," in Proc. 2nd National Conference on Emerging Trends and Applications in Computer Science (NCETACS), pp. 1-4, 2011.

[34] M. Sharif, M. Raza, S. Mohsin, and J. H. Shah, "Microscopic feature extraction method," Int. J. Advanced Networking and Applications, vol. 4, pp. 1700-1703, 2013.

[35] I. Irum, M. Raza, and M. Sharif, "Morphological techniques for medical images: A review," Research Journal of Applied Sciences, vol. 4, 2012.

[36] S. Zhu, X. Xia, Q. Zhang, and K. Belloulata, "An image segmentation algorithm in image processing based on threshold segmentation," in Proc. Third International IEEE Conference on Signal-Image Technologies and Internet-Based System, SITIS'0., pp. 673-678, 2007.

[37] A. Xu, L. Wang, S. Feng, and Y. Qu, "Threshold-based level set method of image segmentation," in Proc. 3rd International Conference 
on Intelligent Networks and Intelligent Systems (ICINIS), pp. 703-706, 2010.

[38] M. Yasmin, M. Sharif, S. Masood, M. Raza, and S. Mohsin, "Brain image enhancement-A survey," World Applied Sciences Journal, vol. 17 , pp. 1192-1204, 2012

[39] W. Kaihua and B. Tao, "Optimal threshold image segmentation method based on genetic algorithm in wheel set online measurement," in Proc. Third International Conference on Measuring Technology and Mechatronics Automation (ICMTMA), pp. 799-802, 2011.

[40] F. Jiang, M. R. Frater, and M. Pickering, "Threshold-based image segmentation through an improved particle swarm optimisation," in Proc. International Conference on Digital Image Computing Techniques and Applications (DICTA), pp. 1-5, 2012.

[41] D. Barbosa, T. Dietenbeck, J. Schaerer, J. D'hooge, D. Friboulet, and O. Bernard, "B-spline explicit active surfaces: An efficient framework for real-time 3-D region-based segmentation," IEEE Transactions on Image Processing, vol. 21, pp. 241-251, 2012.

[42] G. Chen, T. Hu, X. Guo, and X. Meng, "A fast region-based image segmentation based on least square method," in Proc. IEEE International Conference on Systems, Man and Cybernetics, SMC, pp. 972-977, 2009.

[43] Z. Hua, Y. Li, and J. Li, "Image segmentation algorithm based on improved visual attention model and region growing," in Proc. 6th International Conference on Wireless Communications Networking and Mobile Computing (WiCOM), pp. 1-4, 2010.

[44] S. M. M. Sharif, M. J. Jamal, M. Y. Javed, and M. Raza, "Face recognition for disguised variations using gabor feature extraction,"
Australian Journal of Basic and Applied Sciences, vol. 5, pp. 1648-1656, 2011.

[45] M. Sharif, S. Mohsin, M. Y. Javed, and M. A. Ali, "Single image face recognition using laplacian of gaussian and discrete cosine transforms," Int. Arab J. Inf. Technol., vol. 9, pp. 562-570, 2012.

[46] T. Mei, C. Zheng, and S. Zhong, "Hierarchical region based Markov random field for image segmentation," in Proc. International Conference on Remote Sensing, Environment and Transportation Engineering (RSETE), pp. 381-384, 2011.

[47] J. S. M. Sharif, S. Mohsin, and M Raza, "Sub-holistic hidden markov model for face recognition," Research Journal of Recent Sciences, vol. 2, pp. 10-14, 2013.

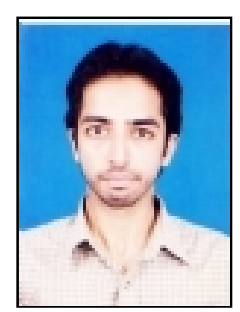

Muhammad Waseem Khan is a student of MS (computer science) at COMSATS institute of information technology, wah campus, Pakistan. He has completed his Bachelor degree from COMSATS Institute of Information Technology, Lahore in 2010. His areas of interest are Image Processing, Information Security and Mobile Computing. 